\title{
CLIL como metodología de enseñanza de inglés mediante música y literatura
}

\author{
CLIL as a Teaching Methodology of the English Language Through Music \\ and Literature
}

\section{CLIL como metodologia de ensino de inglês por meio da música e da \\ literatura}

\author{
Génesis Gabriela Barberán Álvarez \\ Pontificia Universidad Católica del Ecuador, Ecuador \\ ggbarberan@pucesd.edu.ec \\ https://orcid.org/0000-0003-4436-2495
}

Ana Verónica Reza Segovia

Pontificia Universidad Católica del Ecuador, Ecuador

rsav@pucesd.edu.ec https://orcid.org/0000-0002-1707-3930

\section{Resumen}

La enseñanza del inglés como segunda lengua ha ganado un lugar importante en los sistemas educativos del mundo. Por ello, se han desarrollado metodologías buscando que los estudiantes alcancen un aprendizaje significativo de la lengua inglesa. El objetivo del presente trabajo fue aplicar la metodología content language integrated learning (CLIL) a través de la música y la literatura para mejorar el proceso de enseñanza-aprendizaje del inglés de los estudiantes del nivel B1 de la Jordan Language School durante el primer módulo del periodo académico abril-agosto 2020. Se trata de una investigación con un enfoque cuantitativo; un estudio cuasiexperimental. Como parte de los resultados se encontró que el proceso de enseñanza-aprendizaje basado en el método comunicativo enfatiza el conocimiento de gramática y vocabulario para desarrollar las destrezas de producción oral de manera fluida, pero resta intervención a la escritura y lectura. Al aplicar la metodología CLIL mediante la música y literatura, se integra una secuencia de actividades integrales que permite el desarrollo de las cuatro destrezas básicas del idioma, 


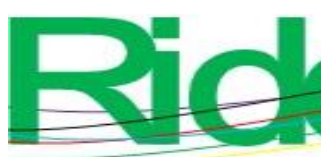

Revista Iberoamericana para la

Investigación y el Desarrollo Educativo

ISSN $2007-7467$

además de dar un valor agregado al incluir contenidos no lingüísticos. A partir de la implementación de CLIL, los estudiantes mejoraron su desenvolvimiento usando el inglés en diferentes contextos sociales, entendiendo las variedades linguiísticas del idioma y produciendo composiciones académicas de forma escrita siguiendo las normas establecidas por el Marco Común Europeo de Referencia para las Lenguas.

Palabras clave: aprendizaje, CLIL, enseñanza, inglés, metodología.

\section{Abstract}

Teaching English as a second language has gained an important place in the educational systems around the world. For this reason, different methodologies have been developed for students to achieve significant learning of the English language. The goal of this work was to apply the content and language integrated learning (CLIL) methodology through music and literature to improve the teaching-learning process of the English language of the students from level B1 of Jordan Language School during the first module of the academic period April-August 2020. It is an investigation with a quantitative approach; a quasi-experimental study. As part of the results, it was found that the teaching-learning process based on the communicative method emphasizes the knowledge of grammar and vocabulary to develop oral production skills in a fluent way, but reduces intervention to writing and reading. By applying CLIL methodology through music and literature, a sequence of integral activities is integrated, which allow the development of the four basic skills of the language, giving added value by including non-linguistic content. From the implementation of CLIL, students improve their performance using English in different social contexts, understanding the linguistic varieties of the language and producing written academic compositions following the standards established by the Common European Framework of Reference for Languages.

Keywords: learning, CLIL, teaching, English, methodology.

\section{Resumo}

O ensino do inglês como segunda língua tem ganhado um lugar importante nos sistemas educacionais do mundo. Por esta razão, metodologias foram desenvolvidas a fim de que os alunos alcancem um aprendizado significativo da língua inglesa. O objetivo deste trabalho foi aplicar a metodologia de aprendizagem integrada de linguagem de conteúdo (CLIL) por meio da música e da literatura para melhorar o processo de ensinoaprendizagem de inglês para alunos do nível B1 da Jordan Language School durante o 


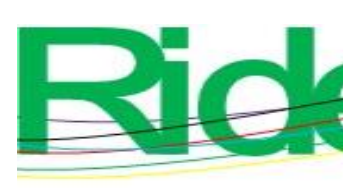

Revista lberoamericana para la

Investigación y el Desarrollo Educativo

ISSN $2007-7467$

Por último, para responder a la interrogante de investigación, se formuló la siguiente hipótesis: la enseñanza del idioma inglés será efectiva a partir de la aplicación de la metodología CLIL.

\section{Materiales y métodos}

La metodología aplicada lleva consigo un enfoque cuantitativo, por cuanto se realizó la recolección de datos a partir del problema identificado y de la revisión de la literatura, lo cual derivó en la formulación de la hipótesis que se sometió a pruebas mediante el empleo del diseño de investigación (Hernández, Fernández y Baptista, 2003).

El estudio es de carácter cuasiexperimental, ya que se investigó el proceso de enseñanza en su contexto real y se limitó al periodo académico quimestral del centro de inglés abril-agosto 2020, donde se recolectaron los datos en diferentes etapas durante las horas de clases.

Se empleó un muestreo por conveniencia. La población de estudio estuvo constituida por nueve estudiantes hombres y mujeres entre 13 y 16 años del nivel B1 de la Jordan Language School de la ciudad de Santo Domingo, Ecuador. Se eligió a este grupo de trabajo debido a que, al estar en un nivel B1 (preintermedio), es más susceptible de llevar la metodología CLIL, ya que los estudiantes no tienen interferencia de contenidos previos en la lengua materna y sus bases en el segundo idioma les permiten entender los nuevos contenidos referentes a las áreas de literatura y música.

Se realizó un diagnóstico de la metodología empleada por el docente. Consistió en la aplicación de una encuesta compuesta por una serie de preguntas cerradas que los estudiantes respondieron con base en las características de las actividades aplicadas durante las clases en comparación con la descripción de las metodologías definidas en cada uno de los ítems de dicha encuesta. Los datos obtenidos permitieron tener un primer acercamiento con el método comunicativo, identificado en la encuesta. Para comprobar la efectividad de estos, se elaboró un plan de clase sobre uno de los temas básicos del nivel B1, el cual incluyó una serie de las actividades más frecuentes a las que los estudiantes han sido expuestos desde que iniciaron su proceso de aprendizaje del idioma inglés (desde los niveles A1 y A2 hasta el B). 
Tabla 1. Plan de clase. Método comunicativo

\begin{tabular}{|c|c|c|c|c|}
\hline \multicolumn{2}{|c|}{$\begin{array}{l}\text { Teacher: MSc. Génesi } \\
\text { Barberán }\end{array}$} & Class: Level B1 & \multicolumn{2}{|c|}{$\begin{array}{l}\text { Institution: Jordan Language } \\
\text { School }\end{array}$} \\
\hline \multicolumn{5}{|c|}{$\begin{array}{l}\text { Objective: Describe the current methodology applied by the English teacher in the } \\
\text { teaching-learning process in order to identify possible problems. }\end{array}$} \\
\hline Stage & & ctivities & Resources & Evaluation \\
\hline Beginning & $\begin{array}{r}\text { Warn } \\
\bullet \\
\bullet \\
\bullet\end{array}$ & $\begin{array}{l}\text { to the students a } \\
\text { of pictures about my } \\
\text { cation and ask them } \\
\text { a verb to represent } \\
\text { ne. } \\
\text { e verbs said by the } \\
\text { ts in order to relate } \\
\text { ry of my vacations } \\
\text { cribing the pictures } \\
\text { one. } \\
\text { torm students asking } \\
\text { vhat they did in their } \\
\text { cations. } \\
\text { song and ask } \\
\text { ts to write and say } \\
\text { verbs in simple past } \\
\text { ey identified. }\end{array}$ & $\begin{array}{ll}\text { - } & \text { Pictures } \\
\text { - } & \text { Computer } \\
\text { - } & \text { Internet } \\
\text { - } & \text { Virtual } \\
& \text { Classroom } \\
\text { - } & \text { Realia }\end{array}$ & $\begin{array}{l}\text { Students will be } \\
\text { evaluated } \\
\text { through: } \\
\text { - Rubric } \\
\text { Scales } \\
\text { - Written } \\
\text { Test }\end{array}$ \\
\hline Construction & $\bullet$ & $\begin{array}{l}\text { tudents the lyrics of } \\
\text { ag and ask them to } \\
\text { at the same time } \\
\text { sten to it. } \\
\text { tudents sang the } \\
\text { explain that they } \\
\text { change the lyrics }\end{array}$ & & \\
\hline
\end{tabular}




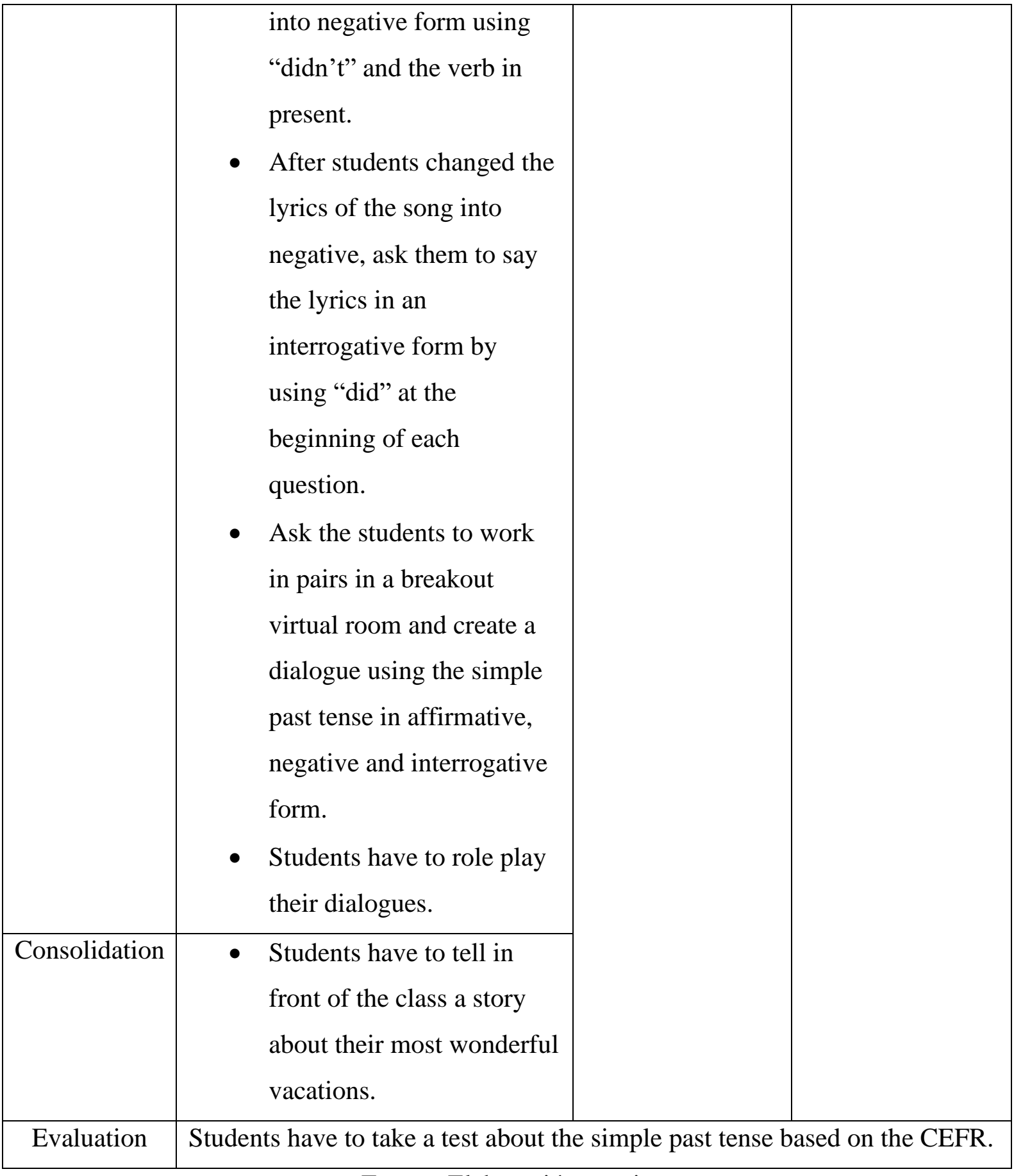

Fuente: Elaboración propia 


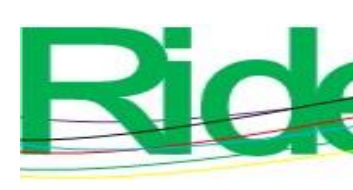

Revista Iberoamericana para la
Investigación y el Desarrollo Educativo
ISSN $2007-7467$

El plan de clase fue ejecutado en tres sesiones. A lo largo de estas se mostraron gráficos relacionados con el tema de "Vacaciones" y se indicó que dijeran un verbo relacionado con cada uno de ellos. Posteriormente se utilizaron los verbos mencionados para contar una historia sobre las vacaciones, lo cual dio paso a realizar una lluvia de ideas sobre las actividades que los alumnos realizaron en sus últimas vacaciones. Se reprodujo una canción y los estudiantes escucharon y escribieron los verbos en tiempo pasado que identificaron en esta; más tarde, se proyectó la letra de la canción para que todo el grupo pudiera cantarla al mismo tiempo que la escuchaba, y al terminar esta cada uno de ellos tuvo que cambiar la letra de afirmativa a negativa y a interrogativa usando los verbos auxiliares did y didn't, respectivamente. Se dividieron las salas de Zoom para que los estudiantes trabajaran en parejas y realizaran un diálogo usando el tiempo gramatical en pasado afirmativo, negativo, interrogativo y respuestas cortas para actuarlo en un role play. Los estudiantes fueron evaluados de manera oral y escrita.

Posterior al diagnóstico y descripción del método comunicativo, se procedió a implementar la metodología CLIL dentro del proceso de enseñanza-aprendizaje del idioma inglés a partir del diseño de un módulo didáctico que se desarrolló durante cinco sesiones de clases, y que incluyó la integración de actividades innovadoras y atrayentes para los estudiantes, las cuales implicaban distintos contenidos relacionados con las áreas literaria y musical (razón por la que se adicionaron dos sesiones más que la metodología anterior sin afectar los resultados de la investigación), además de las actividades propias de la lengua inglesa referentes al mismo tema estudiado en el diagnóstico inicial.

\section{Primera sesión. Introducción}

Se proyectó un video sobre la vida antes y después de Cristo, luego se preguntó a los estudiantes qué les gustaría aprender sobre la historia de la clase y se tomó apuntes de sus opiniones para luego incluirlas en el proceso de enseñanza. Se presentó un mapa de contenidos del módulo y se proyectó un video sobre la historia bíblica de la reina Ester.

\section{Segunda sesión. Investigación}

Se proyectaron imágenes sobre los personajes de la historia de Ester y los estudiantes identificaron a cada uno de ellos diciendo sus nombres. Enseguida, eligieron un personaje e hicieron gestos para que el resto de la clase adivinara quién era y seleccionaron un adjetivo 


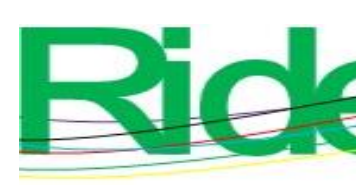

Revista Iberoamericana para la
Investigación y el Desarrollo Educativo
ISSN $2007-7467$

representativo formando una frase con used to (solía). Posteriormente, se proyectaron varias escenas de la historia, los discentes ordenaron cronológicamente y explicaron lo que sucedía en ellas. Para culminar esta actividad, el docente realizó preguntas que los alumnos respondieron con verdadero y falso.

\section{Tercera sesión. Consolidación}

Los alumnos formaron parejas y realizaron un organizador gráfico sobre lo que aprendieron de la historia; además, clasificaron la gramática y vocabulario empleados

\section{Cuarta sesión. Creación I}

Cada estudiante elaboró y expusieron un minilibro digital en el cual resumieron la historia aprendida. Esta actividad dio paso al momento musical: se reprodujo una canción relacionada con la reina Ester para que identificaran las frases que también habían sido usadas en la historia, luego se compartió la letra de la canción para verificar las respuestas y se finalizó esta sesión con un karaoke grupal.

\section{Quinta sesión. Creación II}

Los estudiantes, sin ningún material de apoyo, contaron toda la historia bíblica a la clase haciendo énfasis en su personaje favorito, usando correctamente las estructuras gramaticales, con un amplio vocabulario, fluidez, entonación y pronunciación. Al finalizar las exposiciones, respondieron preguntas que el resto de sus compañeros habían formulado.

Una vez realizadas cada una de las actividades del módulo CLIL, se procedió a evaluar la efectividad de estas mediante la aplicación de una rúbrica de evaluación y un banco de preguntas basados en el Marco Común Europeo de Referencia para las Lenguas (MCERL). Adicional a estos dos instrumentos, se diseñó una prueba para finalmente examinar los contenidos propios de las asignaturas de Música y Literatura. Así, se pudo medir tanto las destrezas del idioma como el conocimiento de las nuevas materias. 


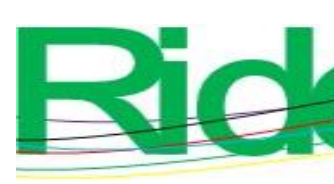

Revista lberoamericana para la Investigación y el Desarrollo Educativo ISSN 2007 - 7467

Figura 2. Calificaciones obtenidas en la destreza de hablar

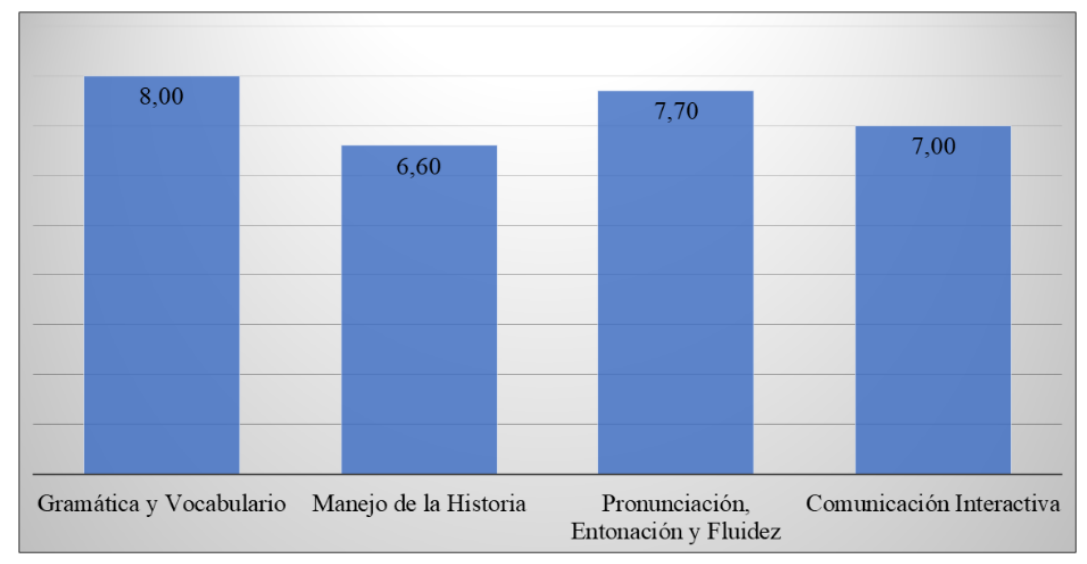

Fuente: Elaboración propia

Los resultados obtenidos en la prueba estandarizada permitieron identificar que los estudiantes presentaron dificultades en la lectura, con una calificación promedio de 5.45, y en la escritura, 7.0 de 10 , como se evidencia en la figura 3.

Figura 3. Calificaciones de gramática, vocabulario, lectura, escucha y escritura

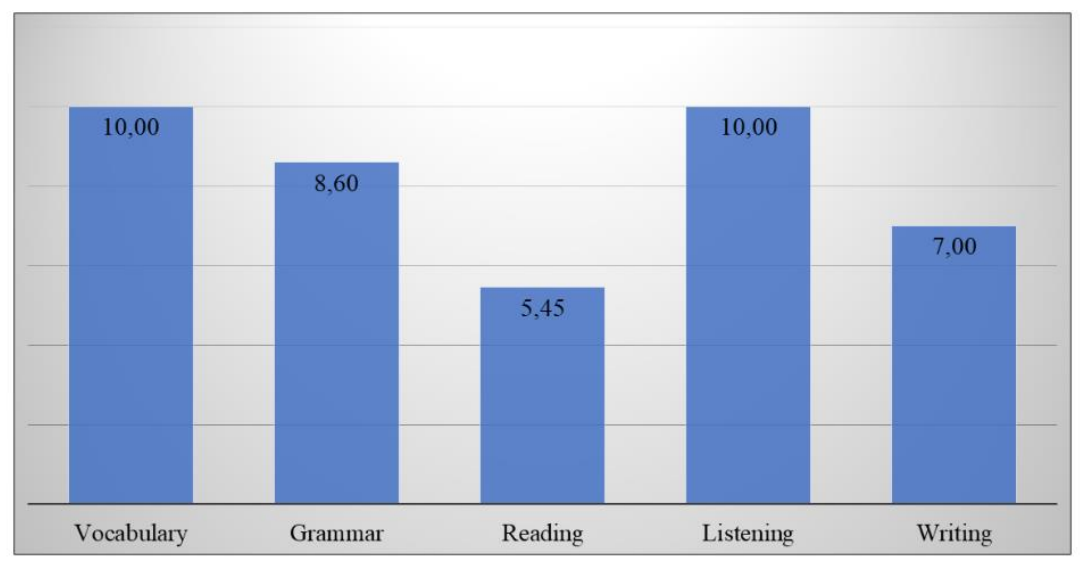

Fuente: Elaboración propia

\section{Implementación de la metodología CLIL para la enseñanza efectiva del idioma inglés a través de las asignaturas de Música y Literatura}

Para la aplicación de esta metodología dentro del centro de inglés ya especificado, se desarrolló un módulo de cinco sesiones, en las cuales se trabajó el mismo tema gramatical que se enseñó a los estudiantes a través del método comunicativo. 


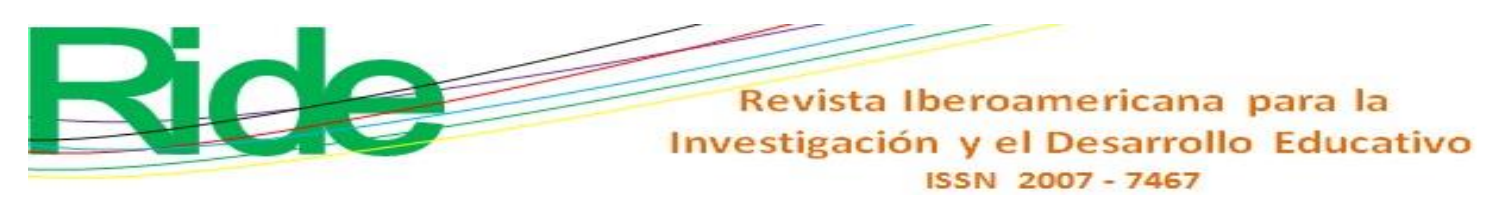

\begin{tabular}{|c|c|c|}
\hline \multirow[t]{4}{*}{ Consolidation } & Spelling & $\begin{array}{l}\text { Ask students to say a value that they identified in } \\
\text { the story. } \\
\text { Write the values on the zoom whiteboard and ask } \\
\text { students to spell them. The student who spells the } \\
\text { words faster is the winner. }\end{array}$ \\
\hline & $\begin{array}{l}\text { Classifying } \\
\text { information }\end{array}$ & $\begin{array}{l}\text { Students have to elaborate a creative graphic } \\
\text { organizer in which they classify the information } \\
\text { that they have learned. }\end{array}$ \\
\hline & $\begin{array}{l}\text { Working in } \\
\text { groups }\end{array}$ & $\begin{array}{l}\text { Distribute students in pairs and make them create } \\
\text { a complete and creative diagram about their } \\
\text { knowledge of the topic. }\end{array}$ \\
\hline & Filling the text & $\begin{array}{l}\text { Students have to complete the test with the } \\
\text { correct grammar structures and appropriate } \\
\text { vocabulary. }\end{array}$ \\
\hline \multirow[t]{4}{*}{ Creation } & Individual work & $\begin{array}{l}\text { Students have to elaborate a digital mini-book in } \\
\text { which they summarize the story and expose them. }\end{array}$ \\
\hline & $\begin{array}{l}\text { Games and } \\
\text { reinforcement } \\
\text { activities }\end{array}$ & $\begin{array}{l}\text { After the expositions, students have to listen to a } \\
\text { song about the story of Esther and after that } \\
\text { underline in the written story the phrases that they } \\
\text { heard. When they finish the activity, they will see } \\
\text { the lyrics of the song and compare their answers. } \\
\text { Finally, students have to sing the song. }\end{array}$ \\
\hline & Presentations & $\begin{array}{l}\text { Without using any supportive material, students } \\
\text { have to tell the story emphasizing a particular } \\
\text { character in front of the class, making use of } \\
\text { grammar correctly, and applying a good } \\
\text { vocabulary, fluency, intonation and } \\
\text { pronunciation. }\end{array}$ \\
\hline & Content test & $\begin{array}{l}\text { Students have to take the test about the content of } \\
\text { the subjects and the language. }\end{array}$ \\
\hline
\end{tabular}




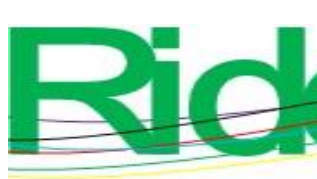

Revista Iberoamericana para la Investigación y el Desarrollo Educativo ISSN $2007-7467$

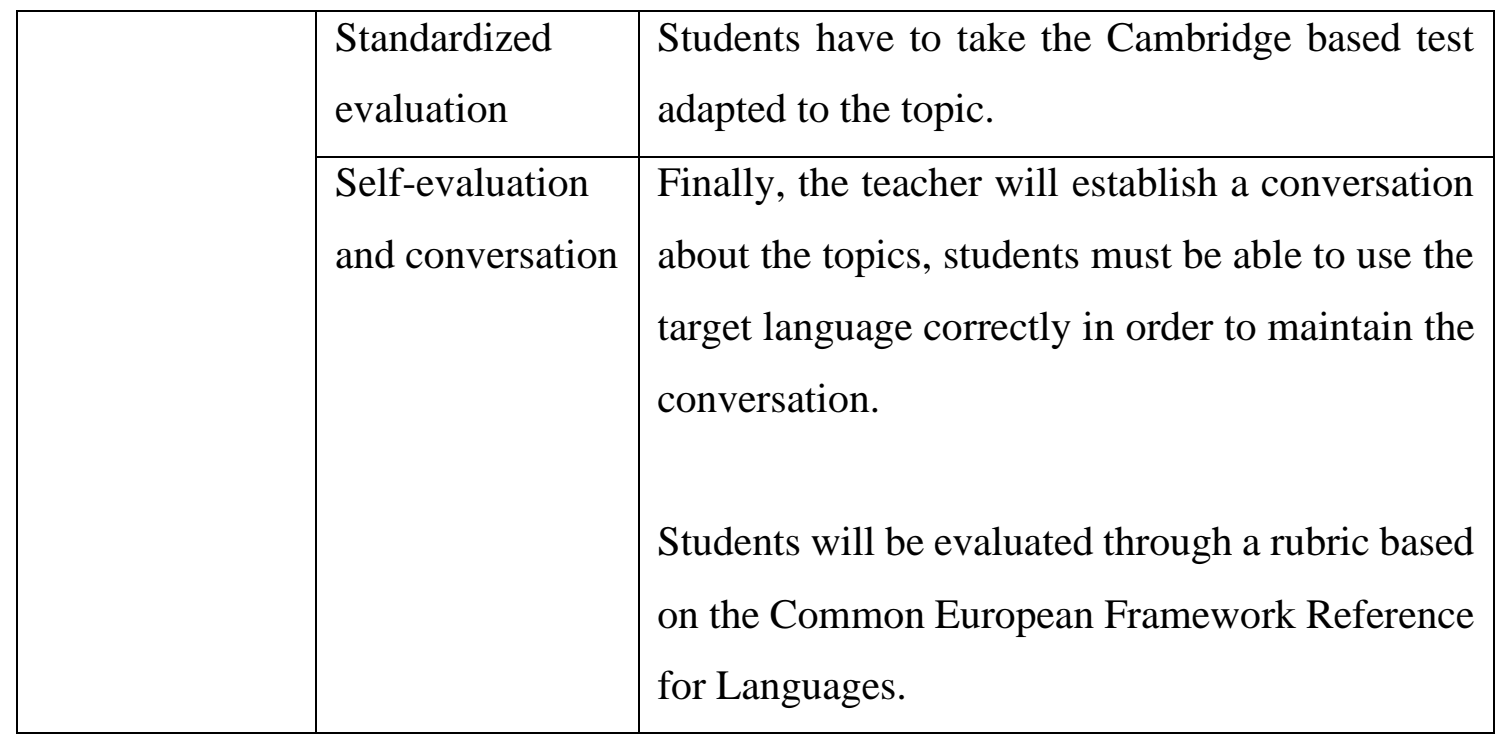

Fuente: Elaboración propia con base en Cano (2013)

\section{Evaluar los resultados que se obtienen a partir de la ejecución de la metodología CLIL para su implementación permanente en el centro de inglés}

Una vez culminadas las actividades desarrolladas a través la metodología CLIL, se evaluaron los resultados obtenidos en cada una de las destrezas del idioma. Los contenidos propios de las asignaturas integradas (Música y Literatura) permitieron fortalecer la práctica que se les dificultó con el método comunicativo: el manejo adecuado de la historia y la interacción con las demás personas, lo cual se reflejó en sus calificaciones: de 6.6 a 8.0 y de 7.0 a 8.0, respectivamente. Esto se evidencia en la figura 4.

Figura 4. Calificaciones obtenidas en la destreza de hablar

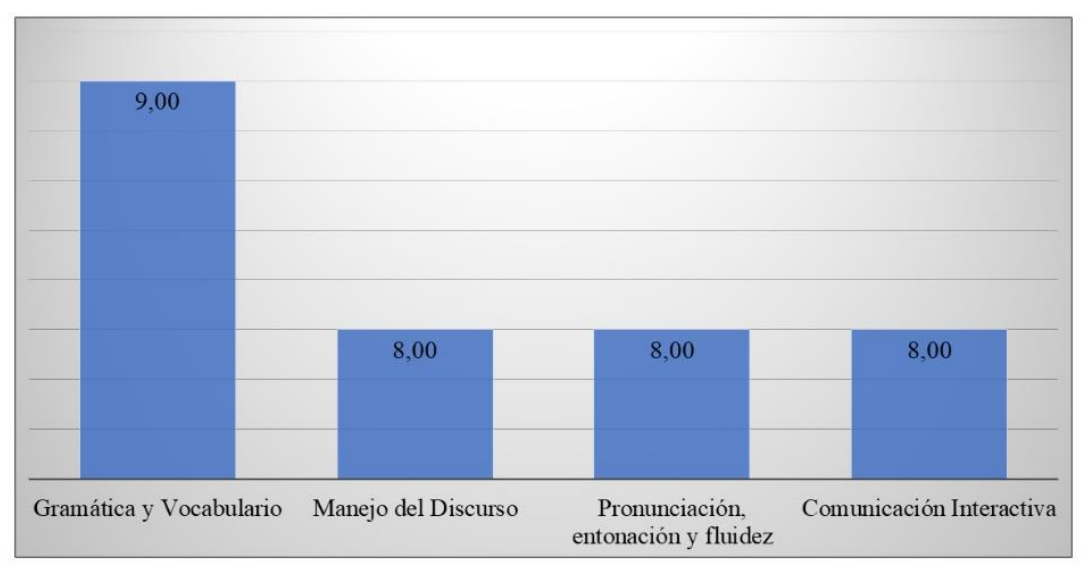

Fuente: Elaboración propia 


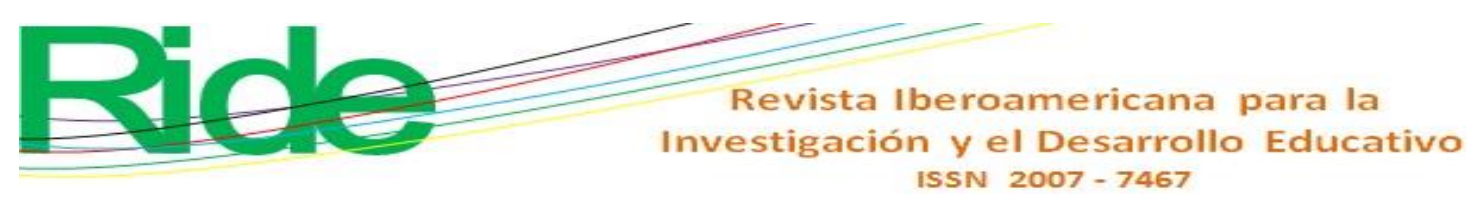

Figura 6. Prueba de contenidos literarios y musicales

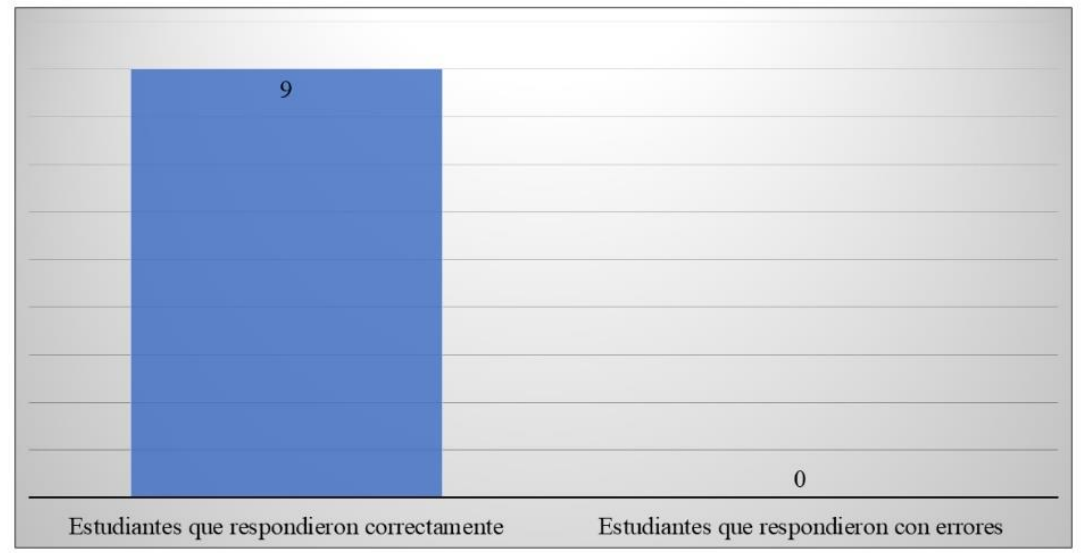

Fuente: Elaboración propia

\section{Discusión}

El método comunicativo tradicionalmente aplicado en el proceso de enseñanzaaprendizaje del idioma inglés en la Jordan Language School permite que los estudiantes desarrollen una base sólida de gramática y vocabulario en su destreza de speaking, aunado a contribuciones relevantes: características fonológicas de las palabras e interacción con muy poca ayuda y apoyo del docente. La destreza de percepción oral, listening, presenta resultados favorables, ya que los estudiantes entienden canciones, diálogos y vídeos interpretados por nativos de la lengua inglesa comprendiendo el contenido, mensaje y enseñanza de estos.

Sin embargo, en la destreza de producción escrita, writing, los estudiantes completan la asignación con una forma limitada del idioma, usando frases repetitivas, y con poca coherencia entre las ideas. En su percepción escrita, reading, no comprenden en su totalidad el mensaje del texto, muestran poco análisis en la asociación de conceptos y definiciones, lo cual demuestra que el método comunicativo hace mayor énfasis en las destrezas orales al buscar que los estudiantes se expresen fluidamente en diferentes situaciones y al enfocarse en ayudarlos a adquirir estructuras gramaticales precisas, pero resta importancia a la lectura y escritura, que son destrezas necesarias para el buen dominio del idioma.

Mediante la implementación y aplicación de la metodología CLIL, los estudiantes desarrollan actividades que fortalecen sus cuatro habilidades del idioma. Durante las presentaciones individuales y grupales, demuestran un buen manejo del idioma: la gramática, vocabulario, pronunciación, entonación, fluidez y comunicación interactiva se muestran de manera natural a través de diálogos, juegos de roles, exposiciones, y presentaciones. En la 


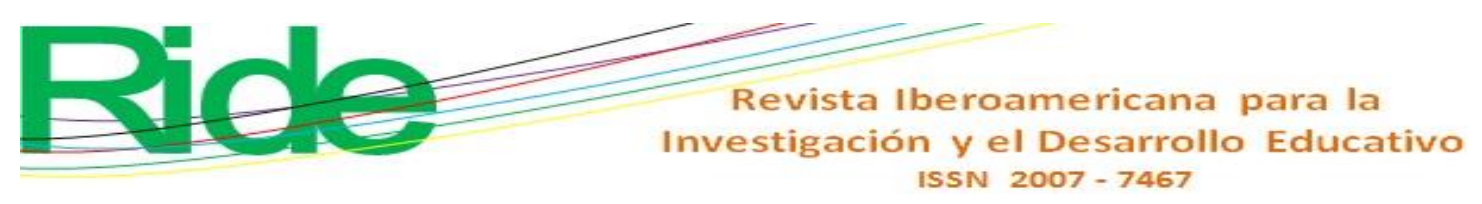

\section{Futuras líneas de investigación}

La presente investigación es de gran impacto para futuros estudios dentro y fuera del país, ya que presenta resultados favorables ante la implementación de una nueva metodología que puede ser aplicada en niños, jóvenes y adultos de todas las instituciones y centros educativos en distintas áreas adicionales a la música y literatura, tales como las ciencias naturales, estudios sociales y matemática, entre otras.

\section{Agradecimientos}

A Jesucristo, mi Dios, porque en todo tiempo Él ha sido bondadoso y Su gran amor me ha permitido alcanzar esta meta; nada sería posible sin Su protección, fortaleza, provisión y sabiduría. Todo proviene de Él, por Él y para Él, ¡a Dios sea la gloria!

A Enrique Barberán y Ángela Álvarez, quienes son el reflejo del amor de Dios por mí, gracias a ellos por ser mis ángeles en la Tierra y mis compañeros de sueños. Ambos han estado siempre junto a mí, guiándome, apoyándome e impulsándome a esforzarme y ser mejor cada día.

A la Pontificia Universidad Católica del Ecuador Sede Santo Domingo, por buscar la excelencia en educación superior y abrir sus puertas para mi formación profesional.

A los estudiantes y padres de familia de la Jordan Language School, gracias por confiar y creer en mí. Ustedes me motivan a prepararme continuamente, actualizarme, innovar y entregar lo mejor de mí.

A todo el personal docente de la Unidad Educativa Palmar del Bimbe por su respaldo y apoyo durante este año de estudio, gracias por su compromiso por mejorar la calidad educativa de nuestra institución.

¿Que Dios llame y guarde sus vidas y prospere sus caminos! 


\section{Referencias}

Abascal, L. (2016). El uso de la literatura en lengua inglesa en el aula de inglés en educación primaria. (trabajo de fin de máster). Universidad de Cantabria, Santander. Recuperado de https://repositorio.unican.es/xmlui/handle/10902/10872.

Cano, W. (2013). Manual CLIL para Centros Bilingües. Logroño, España: Universidad Internacional de la Rioja.

Castro, M. (2014). El uso de la música para la enseñanza del inglés. El Lipdub. (trabajo de fin de grado). Universidad de Valladolid, Soria. Recuperado de https://uvadoc.uva.es/handle/10324/8415

Constitución de la República del Ecuador. (2008). Ciudad Alfaro, Ecuador: Asamblea Constituyente.

Coyle, D., Hood, P. and Marsh (2010). CLIL. Content and Language Integrated Learning. Cambridge, United Kingdom: Cambridge University Press.

Education First. (2019). Informe sobre el nivel de inglés de los hablantes no nativos. Recuperado de https://www.ef.com.ec/epi/regions/latin-america/ecuador/.

Frigols, M. J. (2011). The European Framework for CLIL Teacher Education. Language Teaching, 44(3), 401-402. Retrieved from https://www.researchgate.net/publication/231759242_The_European_Framework_f or_CLIL_Teacher_Education.

Lara, F. y de la Herrán, A. (2016). Reflexiones sobre la educación del Sumak Kawsay en Ecuador. Revista Araucaria, 18 (36), 41-58. Recuperado de https://institucional.us.es/revistas/Araucaria/A\%C3\%B1o\%2020\%20N\%C2\%BA\% 2036\%202016/1.3.\%20Lara\%20Lara.pdf.

Ley Orgánica de Educación Intercultural. (2011). Quito, Ecuador: Asamblea Constituyente. Ministerio de Educación. (2014). Acuerdo 0052-14. Quito, Ecuador: Ministerio de Educación.

Nunan, D. (2003). The impact of English as a global language on educational policies and practices in the Asia-Pacific region. International CLIL Research Journal, 1(2), 415. Retrieved from http://www.icrj.eu/12/article1.html.

Hernández, R., Fernández, C. y Baptista, L. (2003). Metodología de la investigación (5. a ed.). Ciudad de México, México: McGraw-Hill. Recuperado de http://www.pucesi.edu.ec/webs/wp-content/uploads/2018/03/Hern\%C3\%A1ndez- 
Sampieri-R.-Fern\%C3\%A1ndez-Collado-C.-y-Baptista-Lucio-P.-2003.-

Metodolog\%C3\%ADa-de-la-investigaci\%C3\%B3n.-M\%C3\%A9xico-McGraw-

Hill-PDF.-Descarga-en-1\%C3\%ADnea.pdf.

\begin{tabular}{|c|c|}
\hline Rol de Contribución & Autor (es) \\
\hline Conceptualización & $\begin{array}{l}\text { Principal: Génesis Gabriela Barberán Álvarez. IGUAL: Ana } \\
\text { Verónica Reza Segovia }\end{array}$ \\
\hline Metodología & $\begin{array}{l}\text { Principal: Génesis Gabriela Barberán Álvarez. IGUAL: Ana } \\
\text { Verónica Reza Segovia }\end{array}$ \\
\hline Software & $\begin{array}{l}\text { Principal: Génesis Gabriela Barberán Álvarez. IGUAL: Ana } \\
\text { Verónica Reza Segovia }\end{array}$ \\
\hline Validación & $\begin{array}{l}\text { Principal: Génesis Gabriela Barberán Álvarez. IGUAL: Ana } \\
\text { Verónica Reza Segovia }\end{array}$ \\
\hline Análisis Formal & $\begin{array}{l}\text { Principal: Génesis Gabriela Barberán Álvarez. IGUAL: Ana } \\
\text { Verónica Reza Segovia }\end{array}$ \\
\hline Investigación & $\begin{array}{l}\text { Principal: Génesis Gabriela Barberán Álvarez. IGUAL: Ana } \\
\text { Verónica Reza Segovia }\end{array}$ \\
\hline Recursos & $\begin{array}{l}\text { Principal: Génesis Gabriela Barberán Álvarez. IGUAL: Ana } \\
\text { Verónica Reza Segovia }\end{array}$ \\
\hline Curación de datos & $\begin{array}{l}\text { Principal: Génesis Gabriela Barberán Álvarez. IGUAL: Ana } \\
\text { Verónica Reza Segovia }\end{array}$ \\
\hline $\begin{array}{l}\text { Escritura - Preparación del } \\
\text { borrador original }\end{array}$ & $\begin{array}{l}\text { Principal: Génesis Gabriela Barberán Álvarez. IGUAL: Ana } \\
\text { Verónica Reza Segovia }\end{array}$ \\
\hline $\begin{array}{l}\text { Escritura - Revisión y } \\
\text { edición }\end{array}$ & $\begin{array}{l}\text { Principal: Génesis Gabriela Barberán Álvarez. IGUAL: Ana } \\
\text { Verónica Reza Segovia }\end{array}$ \\
\hline Visualización & $\begin{array}{l}\text { Principal: Génesis Gabriela Barberán Álvarez. IGUAL: Ana } \\
\text { Verónica Reza Segovia }\end{array}$ \\
\hline Supervisión & $\begin{array}{l}\text { Principal: Génesis Gabriela Barberán Álvarez. IGUAL: Ana } \\
\text { Verónica Reza Segovia }\end{array}$ \\
\hline $\begin{array}{l}\text { Administración de } \\
\text { Proyectos }\end{array}$ & $\begin{array}{l}\text { Principal: Génesis Gabriela Barberán Álvarez. IGUAL: Ana } \\
\text { Verónica Reza Segovia }\end{array}$ \\
\hline Adquisición de fondos & $\begin{array}{l}\text { Principal: Génesis Gabriela Barberán Álvarez. IGUAL: Ana } \\
\text { Verónica Reza Segovia }\end{array}$ \\
\hline
\end{tabular}

